A N N A L E S Annales de Bretagne et des Pays de l'Ouest

Anjou. Maine. Poitou-Charente. Touraine

109-4 | 2002

Les étrangers dans l'Ouest de la France (XVIII $-\mathrm{XXX}^{\mathrm{e}}$ siècle)

\title{
Les Italiens dans le Morbihan de 1879 à 1939 : un cas de « petite immigration »
}

\section{Bertrand Frélaut}

\section{(2) OpenEdition}

Journals

Édition électronique

URL : http://journals.openedition.org/abpo/1515

DOI : $10.4000 /$ abpo. 1515

ISBN : 978-2-7535-1488-1

ISSN : 2108-6443

Éditeur

Presses universitaires de Rennes

Édition imprimée

Date de publication : 20 décembre 2002

Pagination : $99-112$

ISBN : 978-2-86847-794-1

ISSN : 0399-0826

\section{Référence électronique}

Bertrand Frélaut, «Les Italiens dans le Morbihan de 1879 à 1939 : un cas de « petite immigration » », Annales de Bretagne et des Pays de l'Ouest [En ligne], 109-4 | 2002, mis en ligne le 20 décembre 2004, consulté le 02 mai 2019. URL : http://journals.openedition.org/abpo/1515 ; DOI : 10.4000/abpo.1515 


\title{
Les Italiens dans le Morbihan de 1879 à 1939 : un cas de " petite immigration"
}

\author{
Bertrand FRELAUT \\ Professeur agrégé, lycée Saint-Paul - Vannes \\ membre associé du CHRISCO - Université Rennes 2
}

Pas plus que ses voisins bretons, le département du Morbihan n'est connu pour être une terre privilégiée d'immigration : aux recensements de 1926 ou de 1931, le taux de population étrangère n'est que de $0,05 \%$ et $0,20 \%{ }^{1}$. Des études récentes ont cependant montré l'existence de flux migratoires spécifiques et, notamment, de mosaïstes ou de maçons italiens à Rennes et à Lorient ${ }^{2}$.

L'analyse des demandes de cartes d'identité d'étrangers, imposées par la législation en 1917, a permis de cerner l'immigration italienne en Ille-etVilaine pour les années 1920 et, en tentant la même approche pour le Morbihan, aux Archives départementales, c'est sur un véritable trésor que tombe le chercheur ${ }^{3}$. En passant au crible une cinquantaine de cartons se constitue ainsi un riche corpus de 1389 dossiers individuels, la plupart du temps très documentés, et offrant toute une série de renseignements précis y compris la (ou les) photographie(s) des postulants. Grâce à cette véritable manne héritée d'une administration pointilleuse, mais d'une grande activité, nous pouvons connaître la population italienne dans le Morbihan sous la III ${ }^{\mathrm{e}}$ République, devenue dès 1901 la plus nombreuse parmi les étrangers

1. Arch. dép. du Morbihan, 6M30 et 6M31, soit 314 pour 553175 habitants en 1926 et 1056 pour 536472 en 1931.

2. GuENe, Hélène, Odorico mosaïque art déco, Bruxelles, éditions AAM, 1991, 234 p. ill.; LE BERRE, Rémi, "Lorient méconnu : les maisons de l'entre deux-guerres ", $\operatorname{Ar} M e n, \mathrm{n}^{\circ}$ 20, 1989; PIGEARD DE GURBERT, François, «L'immigration italienne en Ille-etVilaine au début des années 1920 ", Mémoires de la Société d'Histoire et d'Archéologie de Bretagne, tome LXXIV, 1996, p. 217-228.

3. Arch. dép. du Morbihan, Il s'agit des 56 cotes 4M358 à 4M414 où sont classées les demandes d'établissements ou de renouvellements des cartes d'identité d'étrangers faites par les ressortissants italiens auprès de la préfecture du Morbihan de 1917 à 1939. Voir aussi 4M263 à 4M267 contenant des états nominatifs et numériques, des enquêtes et des déclarations de résidence par nationalités. 
du département. On pourra ainsi examiner successivement leur nombre, leur origine géographique, leur classe d'âge, leurs professions, les villes ou les entreprises où ils ont travaillé, voire leur rétribution et leurs conditions d'existence, et la façon dont ils ont pu s'intégrer dans la population locale.

L'importante documentation qui a servi de base à cette étude est parfois disparate. Jusqu'en 1925, on ne dispose que de fiches sommaires constituées d'un seul feuillet, avec photographies et tous les renseignements d'état-civil, mais la législation change à cette date. De 1925 à 1928, la carte d'identité d'étranger est de couleur blanc cassé avec 12 pages pour noter les changements de résidence qui doivent être visés. Dans les années 19291931, elle devient violette et passe à 18 pages. Les dossiers s'étoffent et comprennent des certificats médicaux ou quelques contrats de travail. Les impétrants doivent répondre à des questionnaires de plus en plus précis devant les commissaires de police dans les villes ou les maires dans les communes rurales : commune de résidence, nom, prénom, date et lieu de naissance, noms et prénoms des parents, situation familiale, renseignements sur le conjoint, les enfants de moins de 15 ans, les précédents séjours en France, signature et photographie d'identité. Les fiches cartonnées demandent encore les noms des personnes qui se portent garantes de l'étranger, en Italie ou en France avec leur adresse, des formulaires permettent des vérifications dans les autres départements et, parfois, quelques rapports de police, donnant des informations sur la " moralité " de tel ou tel.

À partir des années 1934, il est exigé des précisions supplémentaires et chaque étranger doit obtenir des cartes de couleurs différents suivant sa situation. Elles se déplient en une vingtaine de rabats destinés à enregistrer les changements de domicile et, leur validité étant limitée à deux ou trois ans, elles doivent être rendues à l'administration pour chaque renouvellement de telle sorte que les dossiers d'italiens sont de plus en plus importants et permettent de suivre l'histoire de chacun (comme d'ailleurs d'observer l'évolution de ses différentes photographies d'identité).

Les cartes sont jaunes pour les travailleurs agricoles, grises pour les artisans, bleues pour les salariés ou travailleurs industriels et vertes pour les non-salariés. Chaque dossier est classé dans une chemise de couleur pour chaque demande d'établissement ou de renouvellement. Cette chemise cartonnée résume les renseignements du dossier et permet d'utiles confrontations. Les naturalisations sont indiquées également. Les années 1920 sont donc moins riches en documentation mais elles correspondent à une faible présence étrangère; les années 1930 sont, au contraire, une source très fiable et, au prix de tri, classement, calculs et recoupements, on peut aboutir à un bilan assez complet.

\section{L'évolution du nombre des immigrés italiens de 1889 à 1940 et la structure par âges}

De 1889 à 1903, le nombre d'étrangers progresse lentement mais régulièrement, passant de 165 à 420 mais, pendant ce laps de temps, ce sont les 
Italiens qui prennent la $1^{\text {re }}$ place à partir de 1901 : de la vingtaine, ils passent à 60 , soit $14,3 \%$, le $1 / 7^{\mathrm{e}}$ du total recensé. Les années $1904-1918$ voient les statistiques globales évoluer de façon peu compréhensible si l'on ne se réfère aux nouvelles règles qui font leur apparition mais la colonie italienne reste toujours la première.

La création de la carte d'identité d'étranger en 1917 donne naissance à de nombreux formulaires grâce auxquels nous sommes désormais bien renseignés et c'est alors que commence véritablement l'essor de l'immigration laborieuse. De 1920 à 1929, la population étrangère double presque, passant de 287 à 513 individus; celle des Italiens sextuple passant de 47 à 281, mais il y a eu un pic dans les années 1924-1927 car grimpé à 785, le chiffre des étrangers est tombé à 514 en 1926 du fait de l'obligation de changer la carte d'identité en carte de travailleur. Les Italiens peuvent alors monter à plus de 50 \% des immigrés de façon un peu artificielle.

Tableau 1 - Les étrangers (dont les Italiens) dans le Morbihan de 1899 à 1941

\begin{tabular}{|c|c|c|c|c|c|c|c|}
\hline & $\begin{array}{c}\text { Population } \\
\text { étrangère totale }\end{array}$ & Dont Italiens & $\%$ & & $\begin{array}{l}\text { Population } \\
\text { étrangère totale }\end{array}$ & Dont Italiens & $\%$ \\
\hline 1889 & 165 & 21 & 12,7 & 1915 & 270 & - & \\
\hline 1890 & - & - & & 1916 & 239 & - & \\
\hline 1891 & 299 & - & & 1917 & 328 & - & \\
\hline 1892 & 302 & - & & 1918 & 380 & - & \\
\hline 1893 & 303 & - & & 1920 & 287 & 47 & 16,3 \\
\hline 1894 & 290 & - & & 1921 & 488 & 100 & 20,4 \\
\hline 1895 & 301 & 46 & 15,3 & 1922 & 561 & 116 & 20,6 \\
\hline 1896 & 297 & - & & 1923 & 640 & 166 & 25,9 \\
\hline 1897 & 295 & - & & 1924 & 757 & 197 & 26,02 \\
\hline 1898 & 312 & 43 & 13,7 & 1925 & 785 & 202 & 25,7 \\
\hline 1899 & 327 & - & & 1926 & 514 & 185 & 58,9 \\
\hline 1900 & 335 & - & & 1927 & 504 & 196 & 38,8 \\
\hline 1901 & 385 & 56 & 14,5 & 1928 & - & - & \\
\hline 1902 & 401 & 56 & 14 & 1929 & 513 & 281 & 54,7 \\
\hline 1903 & 420 & 60 & 14,3 & 1930 & 595 & 342 & 57,4 \\
\hline 1904 & - & - & & 1931 & 1056 & - & \\
\hline 1905 & - & - & & 1932 & - & - & \\
\hline 1906 & 73 & 9 & 12,3 & 1933 & 1094 & 300 & 27,4 \\
\hline 1907 & - & - & & 1934 & - & - & \\
\hline 1908 & 108 & 27 & 25 & 1935 & - & - & \\
\hline 1909 & 109 & - & & 1936 & 1114 & 342 & 30,4 \\
\hline 1910 & 127 & 43 & 33,8 & 1937 & 1244 & 668 & 53,69 \\
\hline 1911 & 130 & 33 & 25,3 & 1938 & 1274 & 676 & 53,06 \\
\hline 1912 & 159 & 38 & 23,9 & 1939 & 1277 & 518 & 40,56 \\
\hline 1913 & 219 & 53 & 24,2 & fin 40 & 996 & 356 & 35,74 \\
\hline 1914 & 260 & - & & & & & \\
\hline
\end{tabular}


Malgré ces nouvelles réglementations, l'immigration reprend dans les années trente. Probablement sous-estimé, le chiffre global passe de 595 en 1930, à 1056 en 1931, pour culminer 1277 en 1939; les Italiens passent, quant à eux, à 342 en 1930 et atteignent leur seuil maximum en 1938 avec 676 représentants, soit $53 \%$ du total. Encore peu nombreux au début du Xxe siècle, ils se sont donc multipliés dans l'entre-deux-guerres de façon tout à fait nette, très au-dessus des moyennes nationales de 29,7 \% en 1931 et 32,8 \% en $1936^{4}$. Les deux derniers chiffres dont nous disposons pour la fin de l'année 1940 et le début de 1941 témoignent d'une vague importante de repli vers la mère-patrie. Les Italiens du Morbihan ne sont plus que 518 en 1939 et 356 à la fin de 1940, sur un effectif total d'étrangers de 996 individus $^{5}$. Même en tenant compte de quelques naturalisations, il y a eu beaucoup de départs "sans esprit de retour", comme le disent les expressions officielles de l'époque portées sur les dossiers.

La composition de cette population par âge et catégorie d'actifs ou non actifs nous est connue à plusieurs dates. Dans les années 1920, le nombre d'hommes qui travaillent évolue de façon irrégulière pour les raisons évoquées ci-dessus.

Tableau 2 - La répartition des hommes sur la population immigrée italienne de 1921 à $1926^{6}$

\begin{tabular}{|c|c|c|c|c|c|c|}
\hline & 1921 & 1922 & 1923 & 1924 & 1925 & 1926 \\
Hommes & 61 & 99 & 139 & 160 & 165 & 116 \\
Total & 100 & 116 & 166 & 197 & 201 & 185 \\
\hline
\end{tabular}

À la fin des années 1930, nous disposons de chiffres donnant la répartition hommes, femmes, enfants.

Tableau 3 - Les hommes, femmes et enfants dans la population immigrée italienne de 1937 à $1939^{7}$

\begin{tabular}{|c|c|c|c|}
\hline & 1937 & 1938 & 1939 \\
\hline Hommes & 352 & 356 & 256 \\
Femmes & 163 & 175 & 133 \\
Enfants & 153 & 145 & 129 \\
Total & 668 & 676 & 518 \\
\hline
\end{tabular}

4. Arch. dép. du Morbihan, 4M264. AmAR, Marianne et MilzA, Pierre, L'immigration en France au XXe siècle, Colin, 1990.

5. Le gouvernement italien a organisé le rapatriement des immigrés à partir de novembre 1938. Voir RAPONE, Léonardo, « Les Italiens en France comme problème de la politique étrangère italienne ", Exils et migrations, les italiens et les espagnols en France 1938-1946, Milza, Pierre et Peschanski, Denis (dir.), L'Harmattan, Bruxelles, 1994.

6. Arch. dép. du Morbihan, 4M263.

7. Arch. dép. du Morbihan, 4M265. 
Le nombre d'hommes baisse donc sensiblement de 1921 à 1938, de l'ordre de $60 \%$ à $48 \%$ du groupe total annuel, traduisant un certain regroupement familial. Sur l'ensemble des 1389 dossiers étudiés, il y a 1065 hommes et 334 femmes, soit 77,68 \% et 23,32\% mais il est difficile d'aller plus loin pour déterminer combien d'hommes sont mariés. Certaines indications permettent de repérer les couples mariés mais plusieurs Italiens ont laissé leur femme au-delà des Alpes. On constate l'existence de familles et parfois d'enfants mais leur relevé est complexe et nécessiterait des investigations et des recoupements qui n'ont pu être réalisés dans le cadre de cette enquête.

Plusieurs couples "mixtes " ont été identifiés, soit un peu plus d'une quarantaine, formés d'un mari italien et d'une femme morbihannaise (ou, parfois, d'un autre département breton), et une trentaine dont la femme est née dans un autre département français. Quand sont indiqués les lieux de mariage et de naissance des enfants, on reconstitue ainsi l'itinéraire géographique et professionnel de la famille. Jusqu'en 1927, la loi spécifie qu'une Française qui épouse un étranger acquiert la nationalité de son mari, sauf à le préciser explicitement. On trouve donc des dossiers de demandes de cartes d'identité d'étranger présentés par des personnes nées et élevées en France, pays qu'elles n'ont jamais quitté. On sursaute parfois en apercevant (à quatre reprises), des photographies de femmes en coiffe bretonne sur ces dossiers d'étrangers...

Après 1927, les épouses gardent leur nationalité d'origine et de nombreuses "réintégrations " sont prononcées. On ne peut donc plus connaître, avec certitude, l'identité des femmes d'immigrés italiens si celles-ci sont françaises puisqu'elles n'ont plus de papier à remplir. Ceci restreint encore notre possibilité d'appréhender de façon satisfaisante, et complète, l'état-civil de nos travailleurs.

Le relevé des âges de naissances permet de réaliser une pyramide des âges des immigrés italiens du Morbihan à partir des années fournies pour 334 femmes et 1000 hommes, ces derniers représentant donc 74,9\% du total. La plupart de ces données concernent les immigrés présents dans le Morbihan dans les années 1930. Chez les femmes, les années 1895-1915, avec 208 références représentent $62 \%$ de l'ensemble. Cette génération est beaucoup plus importante chez les hommes puisque les années 1890-1910, avec plus de 680 sujets, en rassemblant plus de $68 \%$, avec des années particulièrement fortes en 1898 et 1904.

Il s'agit donc de travailleurs dans la force de l'âge - 20 à 50 ans typiques d'une population dont l'exode est, fondamentalement, motivé par des raisons de recherche d'emploi.

Tableau 4 - Répartition des hommes et des femmes selon leur année de naissance

\begin{tabular}{|l|l|l|l|}
\hline \multicolumn{2}{|l|}{ Hommes } & Femmes \\
\hline $1854-1889$ & 215 & $1846-1894$ & 103 \\
$1890-1910$ & 686 & $1895-1915$ & 208 \\
$1911-1924$ & 99 & $1916-1924$ & 23 \\
\hline
\end{tabular}




\section{L'origine géographique des immigrés}

L'analyse des lieux de naissance a donné des résultats pour 1213 dossiers et les constatations sont très nettes et sans ambiguïtés.

Tableau 5 - L'origine géographique des immigrés italiens du Morbihan

\begin{tabular}{|l|c|c|}
\hline Lieux de naissance & nombre & $\%$ \\
\hline Piémont & 233 & $16,7 \%$ \\
Lombardie & 159 & $11,4 \%$ \\
Vénétie & 172 & $12,38 \%$ \\
Frioul & 361 & $25,98 \%$ \\
Ligurie, Émilie, & 70 & $5,03 \%$ \\
Campanie, Sicile, & 47 & $3,38 \%$ \\
Autres régions & 69 & $4,96 \%$ \\
Étranger (dont France) & $102(59)$ & $7,34 \%(4,24 \%)$ \\
\hline
\end{tabular}

Les quatre régions du nord de l'Italie totalisent 66,5\% des immigrants. Un Italien sur deux provient du nord-est (Vénétie-Frioul) et 26 \% d'entre eux sont originaires du Frioul et, particulièrement, des zones montagneuses ou semi-montagneuses de Pordenone, San Daniele del Friuli, Flaibano, Pinzano, Tolmezzo, Fontanafredda, Tramonti di Sopra.

Les mêmes constatations sont valables pour les Piémontais et les Lombards qui viennent des montagnes. C'est par familles entières qu'ils arrivent dans le Morbihan; frères et sœurs, parents et enfants, oncles et neveux et autres apparentés se succèdent, parfois sur trois générations, dont on retrouve les litanies de noms par dizaines : les plus répandus sont les Nadin (13), Brossolo (13), Rossi (11), Picco (11), Ronco (10), Cimolaï (10), Zanier (9), Liva (10), Bertagnolio (9), Facchin (8), Fortini (7), Capraro (7), Galli (6), Regis (6), Della Schiava (6), Grillo (6), etc. ${ }^{8}$.

On comprend assez clairement qu'un flux s'est constitué du nord de l'Italie vers la Bretagne à partir des années 1920, via d'autres départements français au début, la plupart du temps. Si l'on excepte des installations très précoces en Morbihan ou dans l'ouest de la France : les Bergamasco à Ploërmel en 1909, les Ronco à Tours en 1889, à Rennes, en 1899, puis à Vannes, en 1904, les Andreatta à Lorient dès $1891^{9}$, mais sommes toutes assez rares, la majorité commence d'abord à travailler dans la reconstruction des régions de l'Est et du Nord-Est de la France dévastées par la Grande guerre. Beaucoup passent aussi par la région parisienne ou dans des départements de l'Ouest où ils sont embauchés par des compatriotes italiens dont nous retrouvons les certificats dans les dossiers. Dans les années 1930 enfin, ils s'installent en Morbihan dans les entreprises d'ori-

8. Et, avec cinq citations, les Bresin, Bressan, Chiummo, de Cecco, Colombo, Costantino, de Matteo, di Bernardo, Durat ou Lorenzi. Au total, une trentaine de familles représente $14,90 \%$ de nos 1389 dossiers.

9. Ce dernier exemple est donné par Rémi LE BerRe dans Ar Men, cité ci-dessus. 
gine italienne déjà implantées, (quelquefois chez des entrepreneurs locaux) et plusieurs d'entre eux créent leur propre affaire même s'ils s'agit d'une micro-entreprise familiale de deux ou trois personnes. On fait alors venir des jeunes frères ou les enfants laissés au pays, à moins que, la famille ayant déjà fait souche en Morbihan, la deuxième génération soit en passe de trouver aussi de l'emploi sur place.

Cette répartition des immigrés italiens par origine géographique n'est pas tout à fait semblable aux moyennes nationales car, si les régions Nord sont majoritaires, la part du Frioul est nettement plus importante ${ }^{10}$.

\section{Répartition géographique dans le département}

Nous avons pu extraire des indications de lieux de travail ou de résidence dans le Morbihan pour 1302 dossiers sur 1389, ce qui nous donne une statistique riche et précise. Six zones apparaissent très visiblement.

Tableau 6 - Répartition dans le département : résultats globaux

\begin{tabular}{|l|c|c|}
\hline Lorient & 531 & $40,78 \%$ \\
\hline $\begin{array}{l}\text { Environs immédiats de Lorient } \\
\text { (dont Hennebont) }\end{array}$ & $\begin{array}{c}245 \\
(121)\end{array}$ & $18,81 \%$ \\
\hline $\begin{array}{l}\text { Vannes } \\
\text { (Rhuys et Sud-Est) }\end{array}$ & 156 & $11,98 \%$ \\
\hline Auray et littoral de Quiberon-Carnac & 138 & $10,59 \%$ \\
\hline Ploërmel, Guer, Malestroit & 137 & $10,52 \%$ \\
\hline Arrondissement de Pontivy & 95 & $7,30 \%$ \\
\hline
\end{tabular}

Soixante pour cent des Italiens du Morbihan se regroupent dans la région lorientaise, la ville de Lorient elle-même en concentrant plus de 40 \%. L'essentiel des emplois s'y trouvent ainsi qu'à Ploemeur, LarmorPlage, Lanester et Hennebont. Quelques villes accueillent aussi les immigrés : Vannes, Auray, Ploërmel, mais le littoral est plus attractif car on y trouve plus de chantiers qu'à l'intérieur du département, singulièrement au centre et au Nord-Ouest où presque aucune implantation n'est signalée. Cette implantation inégale s'explique donc par les besoins des activités du bâtiment.

Quelques relevés effectués à certaines époques précises corroborent ces constatations de la période 1889-1940.

L'existence de petits chantiers explique l'apparition d'une population italienne dans certaines communes : la construction du barrage de Guerlédan en 1928, du préventorium de Plumelec en 1931, de la mairie de Séné en 1926, des bureaux du Nouvelliste du Morbihan à Lorient en 1928, de l'Hôtel de l'Océan à Saint-Pierre-Quiberon en 1935, des installations du

10. Amar, Marianne et Milza, Pierre, op. cit., p. 189 et Milza, Pierre, Voyage en Ritalie, Paris, Plon, 1993, carte p. 64. 
camp militaire de Coëtquidan en 1930, d'une usine de conserverie à Groix en 1935, du barrage de Kercado, à Carnac, en 1933, etc. Le bâtiment est en effet l'occupation essentielle de nos immigrés.

Tableau 7 - Communes dans lesquelles les Italiens sont les plus nombreux dans les années 1927, 1929, 1930 et fin $1940^{11}$

\begin{tabular}{|l|c|c|c|c|}
\hline & 1927 & 1929 & 1930 & 1940 \\
\hline Lorient & 79 & 112 & 130 & 113 \\
Lanester & - & - & - & 17 \\
Ploemeur et Larmor-Plage & 9 & 17 & 9 & - \\
Hennebont & 16 & 15 & 12 & 22 \\
Vannes & 3 & 30 & 39 & 19 \\
Malestroit & 7 & 3 & 12 & \\
Sarzeau et Saint-Gildas & - & - & - & 22 \\
Auray & 3 & 2 & 4 & - \\
Quiberon et Saint-Pierre-Quiberon & 2 & 14 & 26 & - \\
Pontivy & 6 & 7 & 12 & \\
Guer & - & 6 & 14 & \\
Ploërmel & 20 & 22 & 21 & 9 \\
\hline
\end{tabular}

\section{Les professions des immigrés italiens}

Nous connaissons un peu plus de $72 \%$ des professions des Italiens du Morbihan, chiffre normal car il approche la composition masculine de cette population.

Tableau 8 - Les métiers des immigrés italiens

\begin{tabular}{|l|c|c|}
\hline Métiers & Nombre & Pourcentages \\
\hline Maçons et manœuvres & 248 & $24,8 \%$ \\
Cimentiers & 315 & $31,5 \%$ \\
Carriers, ardoisiers & 125 & $12,5 \%$ \\
Autres métiers du Bâtiment et de l'industrie & 127 & $12,5 \%$ \\
\hline Tertiaire & & \\
$\quad$ - commerce, services & 103 & $10 \%$ \\
$\quad$ - domestiques, religieux, étudiants... & 55 & $4 \%$ \\
\hline Agriculture, mer & 28 & $2 \%$ \\
\hline
\end{tabular}

Le bâtiment occupe 81,3 \% des métiers représentés avec un poids écrasant des maçons salariés (\%) ou des ouvriers cimentiers (31\%) qui ont apporté de leur Frioul ou de leur Vénétie natales leurs savoir-faire et leur tradition dans ce domaine ${ }^{12}$. On signale quelques rares " entrepreneurs "

11. Arch. dép. du Morbihan, 4M266.

12. Voir GuENE, Hélène, op. cit., qui a présenté dans son étude sur les Odorico, les traditions du bâtiment dans le Frioul. 
de ciment mais aussi quelques précisions comme " cimentiers-ravaleurs ", " maçons cimentiers ", " mosaïstes ", quelques apprentis ou " ferrailleurscimentiers ${ }^{13} "$.

Les carriers, tailleurs de pierre, "épinceurs ", ou " mineurs " sont presque tous localisés à la carrière de granit de Polhvern, à Hennebont. Les ardoisiers viennent de la carrière de Cô, à Ploërmel ${ }^{14}$. Les autres métiers du bâtiment les plus fréquemment cités sont les boiseurs-coffreurs, menuisiers, plâtriers, peintres, ou, exceptionnellement, plombier, forgeron, ajusteur, tubiste, mécanicien, électricien.

Il n'est pas surprenant que le secteur primaire agricole et maritime soit peu représenté et rares sont les cas de mareyeurs (2), maraîchers, ou jardinier (4), agriculteurs (une dizaine de personnes à Lanester et Carnac) et domestiques agricoles...

Les métiers du tertiaire ne regroupent qu'à peine $15 \%$ de l'effectif. Les professions libérales sont rarissimes : deux dentistes, un avocat, un compositeur de musique, une artiste de cirque, une chanteuse, un peintre ${ }^{15} \ldots$ Les commerçants et artisans sont limonadiers, restaurateurs, tailleurs d'habit ou ces marchands de tissus ambulants de Naples déjà signalés à la même époque dans les départements voisins ${ }^{16}$. Quelques apprentis (coiffure, pâtisserie, restauration) côtoient quelques " étudiants " principalement ecclésiastiques au noviciat des Pères du Saint-Esprit de Kerlois, à l'abbaye de Langonnet. Des domestiques et " gouvernantes " italiennes sont déclarées chez les religieuses ursulines de Vannes, exilées en Italie de 1908 à $1917^{17}$, chez quelques notables de Lorient, d'Auray, de Vannes (y compris le préfet du Morbihan, M. Jules Scamaroni, en poste de 1933 à 1936).

Signalons enfin des religieuses déclarées comme novices à Vannes, Lorient, Sarzeau et quelques métiers pittoresques : fabricant de statuettes de plâtre, " homme de peine ", " bonne à tout faire ", un capucin originaire d'Istanbul échoué à Lorient, un boucher hippique, un artisan-maître raboteur de parquet, une tenancière de maison de tolérance de Vannes.

Les salaires sont assez variables dans les années 1925-1939 pour lesquelles nous disposons de précisions. Dans la période 1925-1936, un maçon gagne de $2,25 \mathrm{~F}$ à $5,30 \mathrm{~F}$ de l'heure, un cimentier 5 à $6 \mathrm{~F}$, un menuisier ou plâtrier 5,50 à $6,50 \mathrm{~F}$ mais un apprenti maçon ne perçoit que 1,80 $\mathrm{F}$ à 3,50 de l'heure. Certains métiers son très peu payés. Les Italiennes qui sont

13. Les mosaïques se nomment Pierre Candon et Luigi Marini (Lorient) ou les De Cecco, originaires de Murano, près de Venise, et établis à Quiberon.

14. Les épinceurs enlèvent les protubérances des pavés à l'aide de massettes; on signale aussi des " débiteurs de granit".

15. Mario et Madeleine Giraldi, dentistes à Lorient, Castori, avocat près de Vannes, Jean Placide Centanini (originaire de Venise) compositeur de musique qui séjourne à l'l̂le-auxMoines de 1914 à 1917 et dont la femme est artiste lyrique, etc.

16. Ces Napolitains, de la " fraction " de commune de Secondigliano, sont présents aussi en Ille-et-Vilaine en 1920-1921 (F. PIGEARD DE GuRBET, op. cit., p. 226).

17. Elles s'exilèrent d'abord sur les rives du lac Majeur puis à Cassano d'Adda, dans le diocèse de Crémone. 
domestiques chez les Ursulines de Vannes ont $900 \mathrm{~F}$ par mois, nourries et logées; une femme de ménage a $15 \mathrm{~F}$ par jour à Damgan en 1935 mais un journalier agricole italien ne reçoit que $12 \mathrm{~F}$ par jour à Quiberon l'été, en 1937, ou tel autre à Carnac en 1935, 5 F par jour, nourri et logé.

Les écarts se maintiennent dans la deuxième moitié des années trente, après les réformes sociales. Si la plupart des maçons ou cimentiers de la région lorientaise ou des grandes villes est rétribuée à hauteur de 5 à $6 \mathrm{~F}$ de l'heure, faisant donc des journées de 40 F, 48 F, ou même de 55 F (on trouve quelques maxima à 6,70 ou $7 \mathrm{~F}$ de l'heure), il existe des salaires très bas : un maçon à 2,50 F de l'heure à Port-Navalo en 1937, un autre à $3 \mathrm{~F}$ à Damgan en 1936, 3,90 F à Ploërmel et tous les manœuvres et terrassiers à $3 \mathrm{~F}$ ou 3,50 F. Les revenus vont donc du simple au triple et, dans le bâtiment, les rétributions diffèrent suivant qu'on est à Lorient ou dans une petite commune rurale, dans une entreprise importante ou chez un patron de micro-entreprise ${ }^{18}$. On signale même quelques situations très difficiles, à la limite de l'indigence, notamment quand certains immigrés frappés d'une amende pour non-renouvellement de carte de travail, sont incapables de la payer et en sont exemptés après avis du maire ou du commissaire de police ${ }^{19}$.

\section{Accueil, intégration, naturalisations}

Quel accueil ont reçu les immigrés italiens dans le Morbihan et se sontils intégrés à la population? Il semble que, globalement, aucune hostilité ni xénophobie ne se soient manifesté à leur égard sauf dans la région lorientaise chez les artisans locaux du bâtiment ${ }^{20}$.

Les rapports des maires ou des commissaires de police ainsi que les avis des milieux professionnels ou consulaires sont favorables. Le 9 décembre 1938, le commissaire de police de Vannes écrit dans son rapport qu'il y a peu d'étrangers dans la ville, qu'ils ne font pas de politique, que les Italiens ont épousé des Françaises et sont assimilables. Celui de Lorient signale l'existence d'un animateur du mouvement fasciste dans le Morbihan, un entrepreneur de Lorient, agent officieux du consul d'Italie à Nantes. Son influence est peu importante " car beaucoup d'Italiens sont mariés à des Françaises et ils ont perdu de plus en plus l'idée de retourner en Italie ». Le préfet du Morbihan conclut le 20 janvier 1939 : «Rien ne s'oppose à une assimilation rapide des éléments d'origine étrangère introduits

18. Lorient compte une douzaine d'entreprises d'origine italienne à côté de quelques grosses sociétés comme Monteil, Bartissol, Périgaud, Lemaigre, Muller... Ce sont surtout : Bertagnolio, Facchin, Brossolo, Fasola, Chiarovano, Bernasconi. Une quinzaine de communes en abrite plus de vingt dont les plus importantes sont Cimolaï (Hennebont), Bergamasco (Ploërmel), Zochetti (Pontivy), Ronco (Vannes), de Matteo (Lanester).

19. En 1936, un fabricant de statuettes de plâtre et sa famille sont signalés comme indigents à Lorient avec un revenu de $6000 \mathrm{~F}$ par an.

20. LE PAUDER, Anne-Laure, "L'architecture pavillonnaire à Lorient - 1918-1940 ", Arts de l'Ouest. La création bretonne, Rennes, 1995. 
dans l'économie française soit par naturalisation, soit en ce qui concerne les enfants nés en France, par l'effet de la loi ${ }^{21}$."

On voit de nombreux cas d'enfants de familles italiennes scolarisés à l'école publique ou privée (Lorient, Vannes, Pontivy, Arradon); des jeunes gens font leur apprentissage avant d'obtenir un métier. Le fils d'un contremaître-menuisier obtient son certificat d'études primaires en 1931 et est apprenti-dessinateur en 1936; un autre est apprenti-pâtissier ou apprenticoiffeur. Plusieurs sont apprentis-maçons.

Dans les dossiers de naturalisations on compte 137 demandes qui ont été acceptées, des couples pour la plupart, résidant dans le Morbihan au moment de leurs démarches, mais il y a aussi des rejets ou des ajournements. Les nationalités d'origine se répartissent comme suit ${ }^{22}$.

\section{Tableau 9 - Les naturalisations (ou rejets) dans le Morbihan de 1845 à 1940 par nationalités d'origine}

\begin{tabular}{|l|c|c|c|}
\hline & Naturalisations & Rejets & Ajournements \\
\hline Italiens & 84 & 2 & 14 \\
Belges & 10 & 2 & 1 \\
Allemands & 7 & 1 & 2 \\
Polonais & 6 & & \\
Turcs & 5 & 1 & 1 \\
Tchécoslovaques & 4 & & 2 \\
Britanniques & 3 & & 1 \\
Autrichiens & 3 & & \\
Russes & 3 & & 1 \\
Roumains & 2 & & 1 \\
Bulgares & 2 & & \\
Arméniens & 2 & & \\
Luxembourgeois & 1 & & \\
Hongrois & 1 & & \\
Espagnol & 1 & & \\
Portugais & 1 & & \\
Syrien-Égyptien & 1 & & \\
Péruvien & 1 & & \\
Suisses & & & \\
\hline
\end{tabular}

La part des Italiens, 61,31 \% des naturalisations, est donc largement prépondérante, loin devant les Belges (7,3\%), les Allemands (5,10\%) ou les Polonais (4,37\%). Parmi ces 19 nationalités représentées, les latins sont très majoritaires, y inclus les Italiens, avec 64,2 \%, suivi des slaves (11 \%) et des germaniques $(7,3 \%)$.

21. Arch. dép. du Morbihan, 4M265.

22. Arch. dép. du Morbihan, 6M616 à 627; Ces cartons permettent, par recoupement avec les demandes de cartes d'identité, de mieux appréhender le nombre et la composition des familles naturalisées. 
Ces 84 dossiers de naturalisation représentent 64 couples, une douzaine de célibataires et 4 enfants soit un total de 144 personnes ce qui représente $10 \%$ de notre corpus. Parmi les Italiens, 35 des 64 couples naturalisés sont des couples mixtes où la femme est originaire du Morbihan (ou, en minorité d'un autre département). Ce nombre, relativement élevé, explique souvent la demande de naturalisation et la rend plus facile.

Enfin il n'est pas indifférent de noter que, comme sur le plan national, ces naturalisations datent pour les deux tiers des années 1935-1940 et même des années 1930-1940 pour 88 \% des cas. Les années 1937 (11 naturalisations), 1939 (22) et 1941 (11) sont les plus fécondes en ce domaine. La répartition géographique des naturalisation d'Italiens recoupe, en partie, leur implantation dans le département.

\section{Tableau 10 - Les Italiens naturalisés selon leur domicile dans le Morbihan}

\begin{tabular}{|l|l|}
\hline Lorient et ses environs & $\begin{array}{l}40 \\
\text { (dont Lorient : 33) }\end{array}$ \\
\hline Auray et sa région & $\begin{array}{l}10 \\
\text { (dont Presqu'île de Quiberon : 8) }\end{array}$ \\
\hline Vannes et sa région & $\begin{array}{l}15 \\
\text { (dont Vannes : 8) }\end{array}$ \\
\hline Ploërmel, et Nord-Est du Département & $\begin{array}{l}13 \\
\text { (dont Ploërmel : 6) }\end{array}$ \\
\hline Pontivy, Gourin, Le Faouët, Locminé & $\begin{array}{l}6 \\
\text { (dont Pontivy : 3) }\end{array}$ \\
\hline
\end{tabular}

La région de Lorient totalise 47,6 \% des naturalisés (dont Lorient $40 \%$ ); les autres villes ou points forts étant Vannes (9,52\%), la presqu'île de Quiberon (9,52 \%), Ploërmel (7,14 \%), Pontivy et Malestroit (3,50 \% chacune), sinon Guer, Muzillac et la presqu'île de Rhuys. Si Lorient reste en tête, les communes voisines de Lorient sont distancées par des foyers plus isolés du département.

On recense $19 \%$ de rejets ou d'ajournements sur les demandes totales et $14 \%$ pour celles des Italiens. Les raisons n'en sont pas fournies sauf exceptions; elles peuvent être juridiques (liquidation judiciaires), morales (vie " dissipée " ou séparation d'avec une femme restée volontairement en Italie...), mais rarement économiques ou politiques. Voici l'un des seuls avis négatifs, émis d'ailleurs de façon très énergique.

Le maire d'une petite ville de l'intérieur du département écrit que la dame est " paresseuse, violente et buveuse ". Le fils aîné (15 ans) est déjà considéré comme un mauvais sujet, " rapineur et paresseux ». La naturalisation du mari entraînant celle de la femme, " je crois qu'il y a intérêt à rejeter en bloc toutes les demandes, les enfants mal élevés deviendront des éléments malsains dans notre pays ". Le mari est bon ouvrier paraît-il, mais il y en a suffisamment dans la région; la mère ne fréquente que des gens 
tarés; " elle est très suspecte sur le plan politique ", quant à lui, « il ne fait pas de politique pour l'instant mais a fréquenté la cellule communiste de $X$... " (souligné par le maire). "Plutôt l'expulsion que la nationalisation [sic], voilà mon avis très net ". Le préfet du Morbihan transmet le dossier au ministère le 14 février 1940 avec proposition d'ajournement. Une mention manuscrite sur le dossier, " non retrouvé en 1946 ", suggère que cette famille a quitté le Morbihan pendant la guerre.

Pour être peu nombreux par rapport au reste de la France, les Italiens immigrés dans le Morbihan dans la première moitié du Xx ${ }^{\mathrm{e}}$ siècle se distinguent de plusieurs façons. Tout d'abord, leur poids dans le nombre total des étrangers est nettement plus fort, de l'ordre de la moitié plutôt que du tiers, à la fin des années 1930. Une seconde particularité réside dans l'importance des Vénitiens et, surtout, des Frioulans dans la structure géographique des immigrés, soit $12 \%$ et $26 \%$. Ils s'implantent en utilisant des réseaux très organisés. On notera aussi la part prépondérante des emplois du bâtiment ( 81 \%) et, parmi eux, des maçons-cimentiers (53 \%) : c'est une immigration professionnellement très spécifique qui répond à un véritable besoin non couvert par les entreprises locales. Il faut aussi constater que l'implantation géographique des Italiens se calque sur la cartes des villes en privilégiant la région lorientaise (60 \%) mais avec des isolats très marqués tant dans des communes du littoral que du centre Est du département : Quiberon, Sarzeau, Ploërmel, Malestroit, Vannes. Si le taux d'étrangers n'est que de $0,20 \%$ dans le département en 1931, il oscille néanmoins de $0,04 \%$ dans l'arrondissement de Pontivy à $0,11 \%$ dans celui de Vannes et à 0,40\% dans celui de Lorient. En 1936, il est de 0,63\% dans le canton de Lorient II et 1,29 \% dans celui de Lorient I. À Vannes-ville, il atteint 0,60\%.

La dernière originalité de cette micro-migration tient dans son intégration dans la population morbihannaise qui, au travers des naturalisations et des mariages mixtes néanmoins modestes en nombre, a donné au département des entrepreneurs du bâtiment (il en subsiste encore quatre) des élus locaux (un maire de Malestroit) ou diverses personnalités comme un résistant d'Hennebont. Les Italiens n'ont pas formé de communauté à proprement parler avant les années 1950, où l'on voit les Postuans se regrouper et organiser des rencontres et des pèlerinages ${ }^{23}$. On peut donc dire qu'il y a bien eu une greffe qui, pour être modeste, n'en a pas moins été réussie.

23. Milza, Pierre, Voyage en Ritalie, op. cit. 


\section{RESUME}

Le Morbihan ne figure pas dans les départements de forte immigration sous la III ${ }^{\mathrm{e}}$ République, mais on peut y observer les caractères spécifiques de sa principale composante : les Italiens. Au nombre de 300 en 1891, les étrangers atteignent le chiffre de 1277 en 1939 dans un département qui compte alors environ 550000 habitants. Les Italiens, troisième nationalité à l'origine, deviennent les plus nombreux en 1901 avec $15 \%$ des effectifs, et leur poids ne cesse de progresser : $20 \%$ en 1924 et $50 \%$ en 1933. L'étude des demandes de cartes d'identité d'étrangers pour les années 1917-1939 permet de constituer un corpus de près de 1400 personnes et de connaître leur origine géographique, leur âge, leur métier, leur situation familiale, leur lieu de travail dans le département, l'entreprise qui les emploie et, parfois même, leur rémunération ou leur comportement personnel. Un microcosme un peu différent des moyennes nationales.

\section{ABSTRACT}

During the Third Republic Morbihan does not appear as an administrative division with a high immigration rate yet one may notice some specific aspects of its main component: the Italians. The foreigners who were 300 in 1891 reached the number of 1277 in 1939 in an administrative division that counted around 550000 inhabitants then. The Italians - the third nationality at the origin - became the most numerous in 1901, representing $15 \%$ of the total number and it kept increasing: $20 \%$ in 1924 and $50 \%$ in 1933. The application for identity cards from foreigners over the years 1917-1939 help to set up a corpus of around 1400 people and to find out their geographic origins, ages, occupations, marital status, the places where they worked in Morbihan, who they worked for and sometimes even their wages and individual behaviours, thus representing a microcosm slightly different from the national average. 